\title{
FIT interacts with AtbHLH38 and AtbHLH39 in regulating iron uptake gene expression for iron homeostasis in Arabidopsis
}

\author{
Youxi Yuan ${ }^{1,2, *}$, Huilan $\mathrm{Wu}^{1, *}$, Ning Wang ${ }^{1,2}, \mathrm{Jie} \mathrm{Li}^{1}$, Weina Zhao ${ }^{1,2}$, Juan $\mathrm{Du}^{1,2}$, Daowen Wang ${ }^{1}$, Hong-Qing \\ Ling $^{1}$ \\ ${ }^{I}$ The State Key Laboratory of Plant Cell and Chromosome Engineering, National Center for Plant Gene Research, Institute of Genet- \\ ics and Developmental Biology, Chinese Academy of Sciences, Datun Road, Chaoyang District, Beijing 100101, China; ${ }^{2}$ Graduate \\ School of the Chinese Academy of Sciences, Yuquan Road, Shijingshan District, Beijing 100049, China
}

Iron is an essential element for plant growth and development. Iron homeostasis in plants is tightly regulated at both transcriptional and posttranscriptional level. Several bHLH transcription factors involved in iron homeostasis have been identified recently. However, their regulatory mechanisms remain unknown. In this work, we demonstrate that the transcription factor FIT interacted with AtbHLH38 and AtbHLH39 and directly conferred the expression regulation of iron uptake genes for iron homeostasis in Arabidopsis. Yeast two-hybrid analysis and transient expression in Arabidopsis protoplasts showed that AtbHLH38 or AtbHLH39 interacted with FIT, a central transcription factor involved in iron homeostasis in Arabidopsis. Expression of FIT/AtbHLH38 or FIT/AtbHLH39 in yeast cells activated GUS expression driven by ferric chelate reductase (FRO2) and ferrous transporter (IRT1) promoters. Overexpression of $F I T$ with either $A t b H L H 38$ or $A t b H L H 39$ in plants converted the expression of the iron uptake genes FRO2 and IRT1 from induced to constitutive. Further analysis revealed that $F R O 2$ and $I R T 1$ were not regulated at the posttranscriptional level in these plants because IRT1 protein accumulation and high ferric chelate reductase activity were detected in the overexpression plants under both iron deficiency and iron sufficiency. The double overexpression plants accumulated more iron in their shoots than wild type or the plants overexpressing either AtbHLH38, AtbHLH39 or FIT. Our data support that ferric-chelate reductase $F R O 2$ and ferrous-transporter IRT1 are the targets of the three transcription factors and the transcription of $F R O 2$ and $I R T 1$ is directly regulated by a complex of FIT/AtbHLH38 or FIT/AtbHLH39.

Keywords: activation of iron uptake genes, Arabidipsis thaliana, bHLH transcription factor, iron homeostasis, protein-protein interaction

Cell Research (2008) 18:385-397. doi: 10.1038/cr.2008.26; published online 12 February 2008

\section{Introduction}

Iron deficiency is the most common micronutrient deficiency in the world. According to the World Health Organization's estimates, most preschool children and pregnant women in developing countries and at least $30-40 \%$ of preschool age children and pregnant women in developed countries are iron-deficient (http://www.harvestplus.

\footnotetext{
"These two authors contributed equally to this work. Correspondence: Hong-Qing Ling

Tel/Fax: +8610 64860377

E-mail: hqling@genetics.ac.cn

Received 17 January 2008; revised 24 January 2008; accepted 28 January 2008; published online 12 February 2008
}

org/iron.html). Plant foods are the principal iron source for humans. Improving iron content and bioavailability in plant food products would be an efficient and economical method to fight human iron malnutrition. Additionally, iron is also one of the most common factors stunting plant growth and development because it exists mostly in an oxidized form as $\mathrm{Fe}$ (III) in soil, which is not readily available for plants given its low solubility.

Plants have developed mechanisms for effective acquisition of iron from soil. All the dicots and monocots, except members of the Gramineae, use an iron uptake system termed 'strategy I' [1]. The strategy I plants respond to iron-limiting stress by (A) releasing proton and organic acids to acidify the rhizosphere, driving more Fe(III) into solution, (B) reducing Fe(III) to Fe(II) at the root surface 
via Fe(III)-chelate reductase, and (C) transporting Fe(II) across the root epidermal cell membrane via activation of a high-affinity Fe(II)-transport system. There are also morphologic changes in roots, such as formation of transfer cells and increased root hair formation under iron deficiency condition. Iron status is tightly regulated in plants because excess iron can generate hydroxyl radicals and damage cellular constituents such as DNA and lipids [2]

Several genes have been reported for ferric chelate reduction [3-7]. Of them, FRO2 in Arabidopsis is characterized as a major reductase involved in ferric chelate reduction in roots [3]. Transgenic plants overexpressing FRO2 showed better growth under low iron condition compared with wild type plants [8]. Iron-regulated transporters (IRT) are required for transport of ferrous iron across the plasma membrane. Many IRT genes have been identified from various plants, such as IRT1 and IRT2 from Arabidopsis $[9,10]$, LeIRT1 and LeIRT2 from tomato [11], OsIRT1 and OSIRT2 from rice [12,13] as well as PSRIT1 from pea [14]. IRT1 is an essential $\mathrm{Fe}^{2+}$-transporter in Arabidopsis. irt 1 loss of function plants are chlorotic under normal culture condition and can not be complemented by overexpression of IRT2 [15-17]. The expression of IRT1 is controlled at both transcriptional and posttranscriptional levels. Its transcription intensity was enhanced under iron-limiting stress whereas IRT1 protein accumulation quickly diminished once sufficient iron was supplied [18].

FER, encoding a bHLH protein, was the first transcription factor shown to be involved in controlling iron deficiency responses and iron uptake in tomato roots [19]. T3238fer, an insertion mutant of $F E R$, is unable to turn on the iron deficiency responses under iron-deficient stress, exhibits strong chlorosis and dies at early stage under normal culture conditions [19]. Further characterization of T3238fer indicated that FER is involved in controlling the transcription of the ferric-chelate reductase LeFRO1 and the metal transporters LeIRT1 and LeNRAMP1 [20, 5]. AtbHLH29/FIT1/FRU, a functional ortholog of FER in Arabidopsis, has been characterized [21-23] and was recently renamed as FIT ( $\underline{F E R}$-LIKE IRON DEFICIENCY INDUCED TRANSCRIPTION FACTOR [24]). FIT can completely complement the malfunctions of the FER mutant when expressed in T3238fer [23]. The FIT-null mutants fit1-1 and fit1-2 [21] displayed similar iron deficiency symptoms as T3238fer and showed strong growth impairment. The FIT protein is involved in controlling the ferric-chelate reductase FRO2 at transcriptional level and the iron transporter IRT1 at the protein level [21, 22]. However, it is still unknown how FER in tomato and its ortholog FIT in Arabidopsis function in the regulation of iron uptake genes such as those encoding ferric-chelate reductase (FRO2, LeFROI) and the ferrous iron transporter
(IRT1, LeIRT1). Recently, Zhao and Ling [25] observed that the steady state mRNA levels of LeFROI and LeIRT1 were not correlated with the steady state levels of FER mRNA under high $\mathrm{pH}$ culture conditions and suggested that FER might require another unknown factor(s) for controlling the transcription of its target gene(s) under iron starvation.

A. thaliana, a model plant for studying the molecular mechanisms of iron uptake in strategy I plants, possesses a large bHLH family containing 162 members [26]. Apart from FIT (AtbHLH29) which was characterized as a transcription factor responsible for the regulation of iron uptake [21-23], AtbHLH38, AtbHLH39, AtbHLH1OO and AtbHLH1O1 also displayed upregulated transcription in roots and leaves under the condition of iron deficiency [27]. Heterologous ectopic expression of AtbHLH38 and AtbHLH39 in tobacco led to riboflavin synthesis and excretion in transgenic plants [28]. Recently, Ogo et al. [29] showed that OSIRO2, a homolog of AtbHLH38 and AtbHLH39 in rice, is involved in regulation of the response to iron deficiency in rice. In this work, we examined the regulatory mechanisms of three bHLH transcription factors FIT, AtbHLH38 and AtbHLH39 in controlling iron uptake gene expression for iron homeostasis in Arabidopsis. We demonstrated that FIT interacted with AtbHLH38 or AtbHLH39 to directly confer transcription activation of the iron uptake genes FRO2 and $I R T 1$. Overexpression of AtbHLH38 or AtbHLH39 with FIT in Arabidopsis changed the expression pattern of $F R O 2$ and IRTI from induced to constitutive activation. More significantly, these transgenic plants were more tolerant of iron deficiency and accumulated more iron in their shoots.

\section{Results}

FIT interacts with AtbHLH38 or AtbHLH39 and confers transcriptional activation of FRO2 and IRT1 in yeast cells

FIT, encoding a bHLH protein, is a key transcription factor involved in regulating the expression of iron uptake genes FRO2 and IRT1 in Arabidopsis [21, 23, 24]. Overexpression of FIT did not change the expression patterns of $F R O 2$ and $I R T 1$ [21], suggesting that FIT may function as a heterodimer in the regulation of its target genes. To examine the effect of FIT in regulating iron uptake, we performed microarray analysis of RNAi plants with down-regulated FIT expression [30]. Analysis of the microarray data revealed that the steady state mRNA level of At3g56980 was significantly elevated under normal culture condition. At3g56980 encodes a bHLH protein, termed AtbHLH39 [31]. BlastX search showed that AtbHLH39 shared high sequence identity with At3g56970 (AtbHLH38), which is not present on the Affymetrix chip. The two genes are 
located in tandem on chromosome 3. Further analysis with the deficiency stress of either $\mathrm{Fe}, \mathrm{Zn}$ or Mn revealed that the expression of AtbHLH38 and AtbHLH39 was induced in shoots and roots when plants were grown under iron deficiency, while no or very low expression was detected in the leaves and roots of plants grown under $\mathrm{Zn}$ or $\mathrm{Mn}$ deficiency conditions (Supplementary information, Figure S1A-S1D). Consistent with the observations recently reported by Wang et al. [27] and Vorwieger et al. [28], the expressions of AtbHLH38 and AtbHLH39 were regulated by iron deficiency (Supplementary information, Figure S1E and S1F).

Based on their specific expression pattern, we hypothesized that AtbHLH38 and AtbHLH39 are possible candidates for dimerization with FIT in Arabidopsis in regulating iron uptake. We first examined whether FIT interacted with AtbHLH38 or AtbHLH39 by yeast two-hybrid assay. FIT was fused to the $\mathrm{C}$-terminus of the activation domain of
GAL4, and AtbHLH38 and AtbHLH39 were separately fused to the C-terminus of the GAL4 DNA-binding domain. An interaction between these fusion proteins would allow yeast cells to grow on synthetic minimal (SM) medium lacking histidine and activate the expression of $\beta$-galactosidase in yeast cells. Two transformed yeast strains harboring plasmids pAD-FIT/pBD-AtbHLH38 and pADFIT/pBD-AtbHLH39 grew on histidine-free SM plates, whereas the strains with other plasmid combinations did not grow on this plate (Figure 1A). Filter lift assays showed $\beta$-galactosidase activity in the two strains with plasmids pAD-FIT/pBD-AtbHLH38 and pAD-FIT/pBD-AtbHLH39, but not in the strains with other plasmid combinations (Figure 1B). For determination of the specificity of the interactions, AtbHLH4O was used as a negative control in this experiment. The yeast strain harboring $\mathrm{pAD}-F I T / \mathrm{pBD}-$ AtbHLH4O hardly grew on SM plate lacking histidine, and showed no $\beta$-galactosidase activity (Figure 1A and 1B).
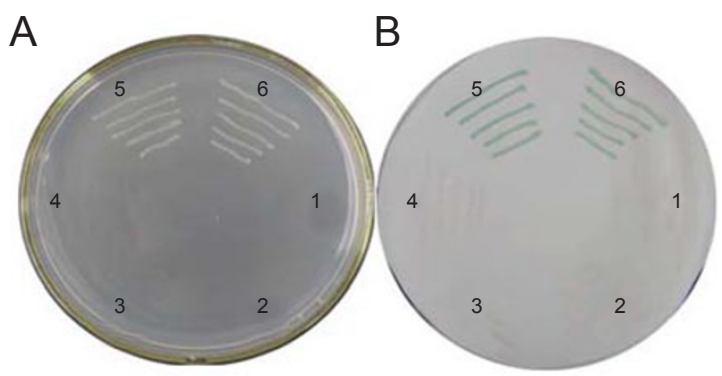

C
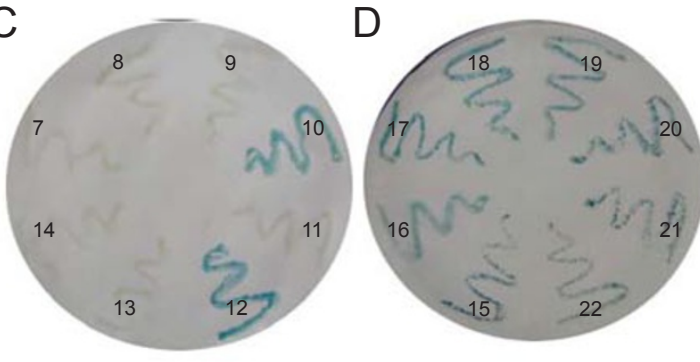
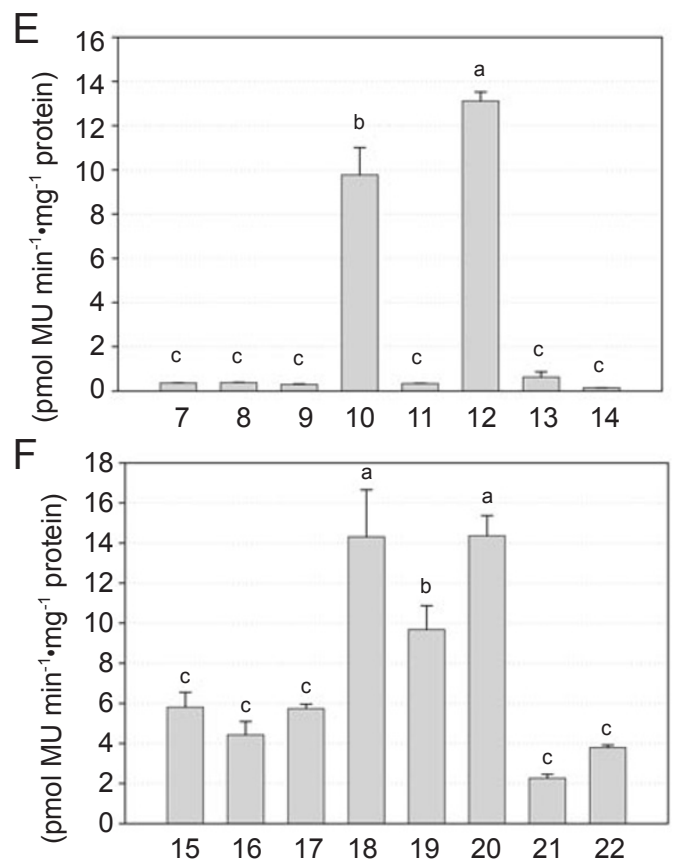

Figure 1 Interaction analysis of FIT with AtbHLH38 and AtbHLH39 by yeast two-hybrid (A, B) and assaying transcriptional activation of GUS expression driven by FRO2 and IRT1 promoters in yeast cells (C-F). The numbers of 1 to 22 indicate plasmid combinations as follows: 1 pAD-FIT/pBD-GAL4, 2 pAD-GAL4/pBD-AtbHLH38, 3 pAD-GAL4/pBD-AtbHLH39, 4 pAD-FIT/pBD-AtbHLH40,

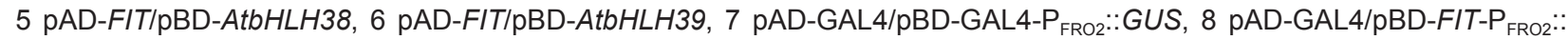

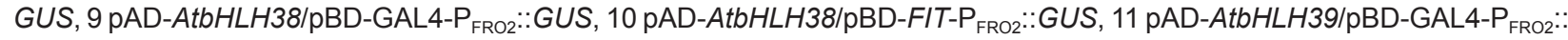

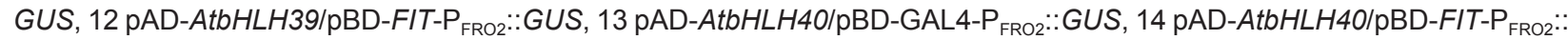
GUS, 15 pAD-GAL4/pBD-GAL4-P $\mathrm{IRT}_{1}:: G U S, 16$ pAD-GAL4/pBD-FIT-P $\mathrm{IRT}_{1}:: G U S, 17$ pAD-AtbHLH38/pBD-GAL4-P IRT1 $:: G U S, 18$ pAD-AtbHLH38/pBD-FIT-P IRT $1_{1}:$ GUS, 19 pAD-AtbHLH39/pBD-GAL4-P ${ }_{\text {IRT } 1}:: G U S, 20$ pAD-AtbHLH39/pBD-FIT-P ${ }_{\text {IRT } 1}:: G U S, 21$ pAD-AtbHLH4O/pBD-GAL4-P $\mathrm{IRT}_{1}:: G U S, 22$ pAD-AtbHLH4O/pBD-FIT-P $\mathrm{IRT}_{\mathrm{IR} 1}:: G U S$. (A) Histidine prototrophy assay on histidinefree SM plate. (B) Filter lift assay of X-Gal activity of yeast strains grown on SM plate with histidine. (C-D) Qualitative analysis of GUS expression driven by FRO2 ( $\left.\mathrm{P}_{\mathrm{FRO} 2}\right)$ and IRT1 $\left(\mathrm{P}_{\mathrm{IRT} 1}\right)$ promoters in the yeast strains grown on SM plates with histidine. (E-F) Quantitative analysis of the GUS expression driven by $P_{F R O 2}$ and $P_{I R T 1}$ in yeast strains. The results in (E-F) are means of 3 independent experiments. Different letters indicate significant differences $(P<0.05)$ among the strains. 
These data demonstrated that the interaction of AtbHLH38 or AtbHLH39 with FIT in yeast cells is specific.

To examine whether AtbHLH38 and AtbHLH39 directly interact with FIT in regulating iron uptake genes such as FRO2 and IRTI in Arabidopsis, we designed a yeast assay system. The FRO2 promoter (947 bp upstream of FRO2 start codon, named as $\mathrm{P}_{\mathrm{FRO} 2}$ ) and $I R T 1$ promoter (sequence from $18 \mathrm{bp}$ to $1598 \mathrm{bp}$ upstream of the IRTI start codon, named as $\mathrm{P}_{\mathrm{IRT}}$ ) were separately fused with the reporter gene GUS for construction of the expression cassettes $\mathrm{P}_{\mathrm{FRO} 2}$ :: GUS and $\mathrm{P}_{\mathrm{IRT}}:: G U S$. The expression cassettes were then cloned into the PmacI site between $\mathrm{fl}$ origin and TRP1 of pBD-WT yeast expression vector and transformed into yeast strain YRG-2. Histochemical assay showed that no GUS activity was detected in yeast strains containing the plasmid $\mathrm{pBD}-\mathrm{GAL} 4-\mathrm{P}_{\mathrm{FRO} 2}:: G U S$, indicating that the expression cassette of $\mathrm{P}_{\mathrm{FRO} 2}: \because G U S$ can not be activated by yeast transcripton (Figure 1C). The yeast strains containing the plasmid with the $\mathrm{P}_{\mathrm{IRT} 1}:: G U S$ expression cassette exhibited weak GUS activity, implying that the IRT1 promoter of Arabidopsis could be activated by the yeast transcription machinery (Figure 1D).

To test whether the transcription factors FIT, AtbHLH38 and AtbHLH39 are able to directly activate the transcription of the major iron uptake genes $F R O 2$ and $I R T 1$, the coding sequences of AtbHLH38 and AtbHLH39 were cloned at EcoRI/SalI site of pAD-WT, while the coding sequence of FIT was inserted at EcoRI/SalI site of pBD-GAL4-P $\mathrm{FRO}_{2}:$ : GUS and pBD-GAL4- $\mathrm{P}_{\mathrm{IRT} 1}: \because G U S$ to generate yeast expression plasmids pAD-AtbHLH38, pAD-AtbHLH39, pBDFIT- $\mathrm{P}_{\mathrm{FRO} 2}:: G U S$ and $\mathrm{pBD}-F I T-\mathrm{P}_{\mathrm{IRT} 1}:: G U S$. As a negative control, the coding sequence of AtbHLH4O was also cloned at EcoRI/SalI site of pAD-WT to produce plasmid pADAtbHLH40. The plasmids were introduced individually and in pairs into the yeast strain YRG-2. Filter lift assays for GUS staining showed that strong blue color appeared in yeast cells containing plasmids pAD-AtbHLH38/pBD-FIT$\mathrm{P}_{\mathrm{FRO} 2}:: G U S$ and $\mathrm{pAD}-A t b H L H 39 / \mathrm{pBD}-F I T-\mathrm{P}_{\mathrm{FRO} 2}:: G U S$, whereas no blue color was observed in the yeast cells with other plasmid combinations (Figure 1C). Consistent with the results of filter lift assays, the yeast cells containing the plasmid combinations of pAD-AtbHLH38/pBD-FIT$\mathrm{P}_{\mathrm{FRO} 2}:: G U S$ and $\mathrm{pAD}-A t b H L H 39 / \mathrm{pBD}-$ FIT-P $\mathrm{P}_{\mathrm{FRO} 2}:: G U S$ exhibited strong GUS activity (Figure 1E). These results clearly showed that the interaction of FIT with AtbHLH38 or AtbHLH39 activated the expression of GUS driven by the $F R O 2$ promoter $\mathrm{P}_{\mathrm{FRO} 2}$ in yeast cells.

As the yeast transcripton can activate the expression of $\mathrm{P}_{\mathrm{IRT} 1}:: G U S$ in yeast cells, GUS activity was observed in all yeast strains containing $\mathrm{P}_{\text {IRT1 }}:: G U S$ in the filter lift assay (Figure 1D). However, the GUS activity in yeast cells containing plasmids pAD-AtbHLH38/pBD-FIT-
$\mathrm{P}_{\mathrm{IRT}}:: G U S$ and $\mathrm{pAD}-A t b H L H 39 / \mathrm{pBD}-F I T-\mathrm{P}_{\mathrm{IRT}}:: G U S$ are significantly higher than that with other plasmid combinations (Figure 1F), suggesting that the combinations of FIT with AtbHLH38 or AtbHLH39 are able to enhance GUS expression driven by the $\mathrm{P}_{\text {IRT1 }}$ promoter in yeast cells. In addition, higher GUS activity was detected in yeast cells containing plasmids pAD-AtbHLH39 and pBD-GAL4$\mathrm{P}_{\mathrm{IRT}}:: G U S$ (Figure $1 \mathrm{~F}$ ).

Interaction of FIT with AtbHLH38 or AtbHLH39 in Arabidopsis cells

To further confirm the protein/protein interaction of FIT with AtbHLH38 or AtbHLH39 in Arabidopsis cells, the bimolecular fluorescence complementation (BiFC) assay system was used [32]. The coding sequences of FIT, AtbHLH38 and AtbHLH39 were amplified with gene-specific primers (Supplementary information, Table S1) and cloned into $\mathrm{pX}$-nYFP and $\mathrm{pX}$-cCFP vectors to generate constructs of BiFC-FIT-nYFP, BiFC-FIT-cCFP, BiFC-AtbHLH38nYFP, BiFC-AtbHLH38-cCFP, BiFC-AtbHLH39-nYFP and $\mathrm{BiFC}-A t b H L H 39-c \mathrm{CFP}$. These constructs were introduced in pairs into Arabidopsis protoplasts by PEG transformation. After transient expression for $18-24 \mathrm{~h}$, the protein/protein interactions were determined by confocal microscopy. A strong YFP signal was detected in nuclei of protoplasts transformed with the plasmids BiFC-FIT-nYFP/BiFCAtbHLH38-cCFP (Figure 2B), BiFC-FIT-nYFP/BiFCAtbHLH39-cCFP (Figure 2C) and BiFC-FIT-nYFP/ BiFC-FIT-cCFP (Figure 2D), whereas no YFP signal was observed in protoplasts transformed with constructs $\mathrm{BiFC}$ AtbHLH38-nYFP/BiFC-AtbHLH38-cCFP (Figure 2E), BiFC-AtbHLH38-nYFP/BiFC-AtbHLH39-cCFP (Figure 2F) and BiFC-AtbHLH39-nYFP/BiFC-AtbHLH39-cCFP (Figure $2 \mathrm{G}$ ), as well as with the empty vectors $\mathrm{pX}$-nYFP/ $\mathrm{pX}$-cCFP (Figure 2H). These results clearly illustrate that FIT interacts with AtbHLH38 or AtbHLH39 in plant cells, while no interaction was observed between AtbHLH38 and AtbHLH39. In addition, FIT showed an interaction with itself (Figure 2D) whereas such self-interactions did not occur with AtbHLH38 and AtbHLH39 (Figure 2E and 2G). Consistent with that FIT, AtbHLH38 and AtbHLH39 are transcription factors, their interaction signals were clearly localized in the nucleus (Figure 2B-2D), whereas the control YFP protein was distributed throughout the cell (Figure 2A).

The loss of AtbHLH38 or AtbHLH39 function did not affect the transcription of FRO2 and IRT1

To examine whether loss-of-function in AtbHLH38 or AtbHLH39 affects the transcription of iron uptake genes, T-DNA insertion lines of AtbHLH38 (salk_108159, hereafter named $m 38$ ) and AtbHLH39 (salk_025676, hereafter 

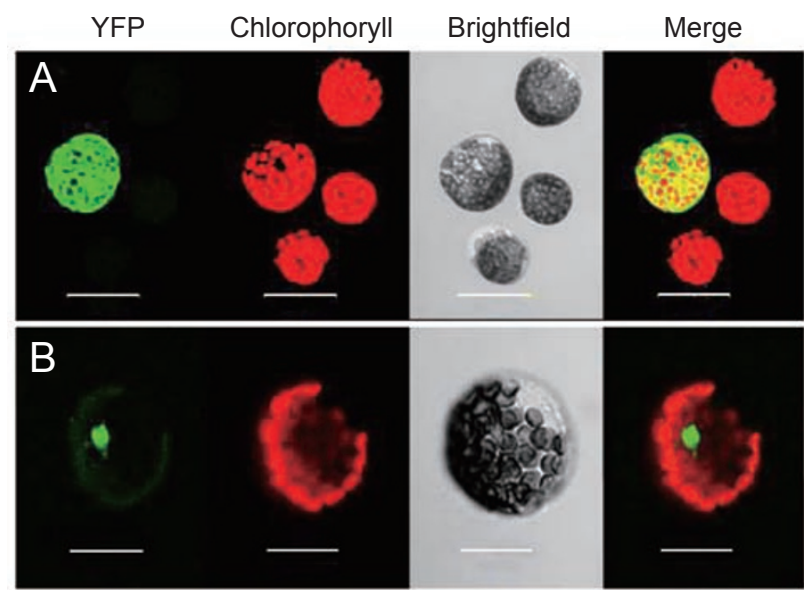

pA7-YFP

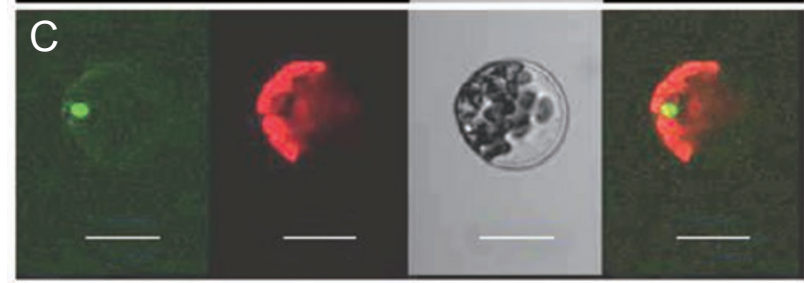

\section{FIT-nYFP+}

AtbHLH38-cCFP

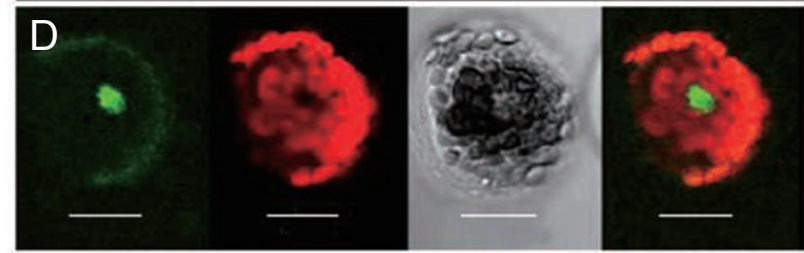

FIT-nYFP+

FIT-CCFP

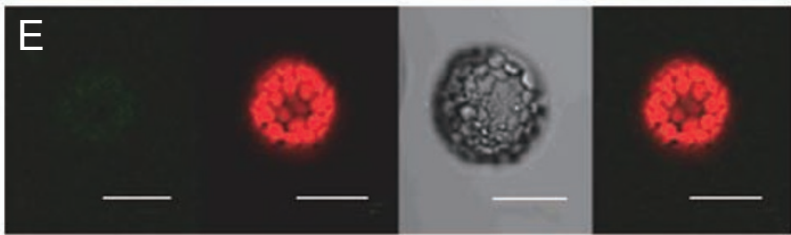

FIT-nYFP+

AtbHLH39-cCFP

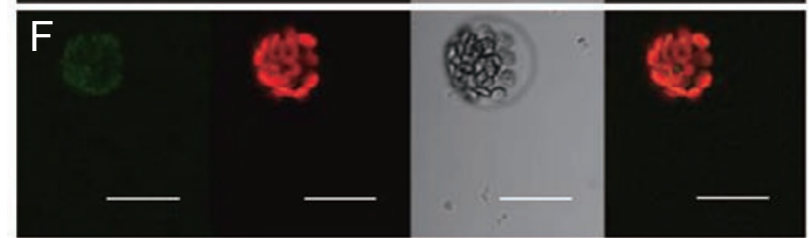

AtbHLH38-nYFP+

AtbHLH38-cCFP

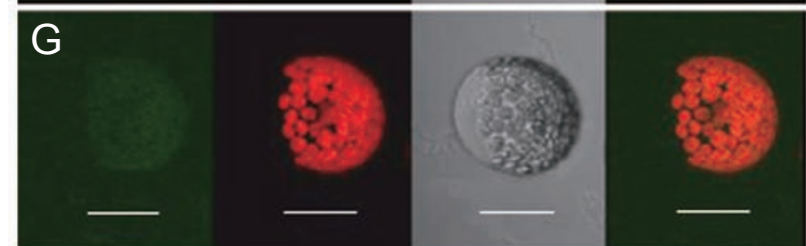

AtbHLH39-nYFP+

AtbHLH39-cCFP

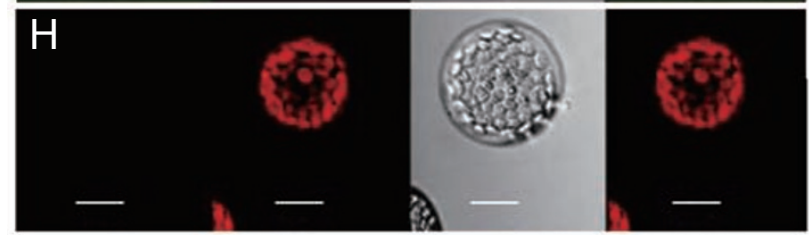

AtbHLH38-nYFP+

AtbHLH39-cCFP

pX-nYFP+

$p X-c C F P$

Figure 2 BiFC assay of protein/protein interaction of FIT, AtbHLH38 and AtbHLH39 in Arabidopsis cells. Images were captured $20 \mathrm{~h}$ after transient expression under an Olympus confocal microscope. Bar=20.0 $\mu \mathrm{m}$. 
termed $m 39$ ) were analyzed together with fit1-2, a T-DNA insertion line of FIT [21, 22]. It was shown that the expression of AtbHLH38 and AtbHLH39 was completely knocked out in $m 38$ or $m 39$ plants (Supplementary information, Figure S2). However, $m 38$ and $m 39$ exhibited a normal growth pattern as wild type both on iron-limited MS plates and in soil, whereas fit1-2 displayed chlorosis and strong growth restriction under the same conditions (data not shown). These results are consistent with the report by Wang et al. [27], indicating that disruption of AtbHLH38 or AtbHLH39 did not lead to phenotypic changes in the plants.

To further investigate whether loss of AtbHLH38 or AtbHLH39 expression affects the expression patterns of the iron uptake genes FRO2 and IRT1, the steady state mRNA levels of the two genes were analyzed by Northern blotting analysis. Consistent with previous reports [21], loss of FIT expression prevented induction of FRO2 and dramatically down-regulated the mRNA accumulation of IRT1 under iron-limiting stress compared to wild type (Figure 3 ). However, no significant differences in FRO2 and IRT1 mRNA abundances were observed in the iron-deficient roots of $m 38$ or $m 39$ in comparison with wild type, demonstrating that knocking out one of the two genes did not affect the transcription of FRO2 and IRT1.

Transgenic plants expressing FIT/AtbHLH38 or FIT/AtbHLH39 are more tolerant of iron deficiency and accumulate more iron in their shoots

Co-expression of FIT together with AtbHLH38 or AtbHLH39 is able directly to activate transcription of FRO2 and IRTI

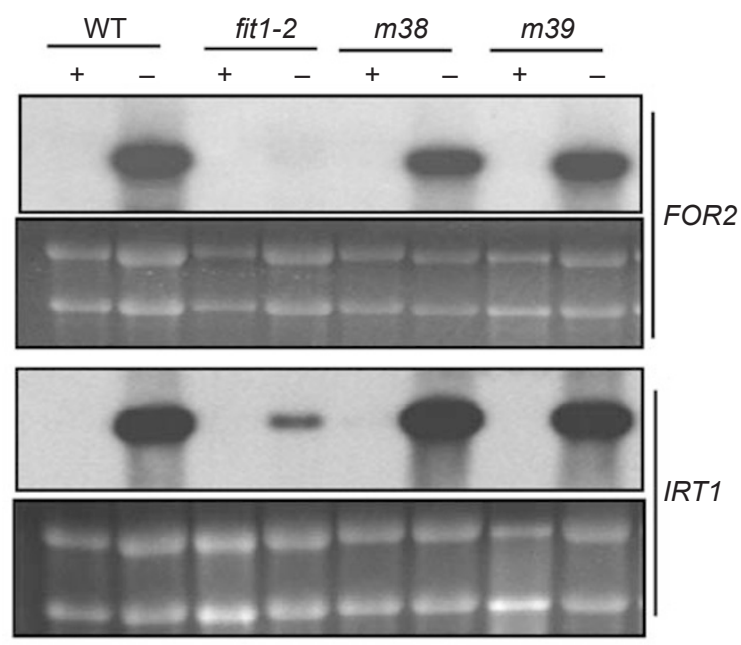

Figure 3 Northern blot analysis of FRO2 and IRT1 in the T-DNA insertion lines fit1-2, $m 38$ and $m 39$. WT: wild type; fit1-2, $m 38$ and m39: T-DNA insertion mutant of FIT, AtbHLH38 and AtbHLH39, respectively; - and + sign indicate the plants grown under the conditions without and with iron, respectively.
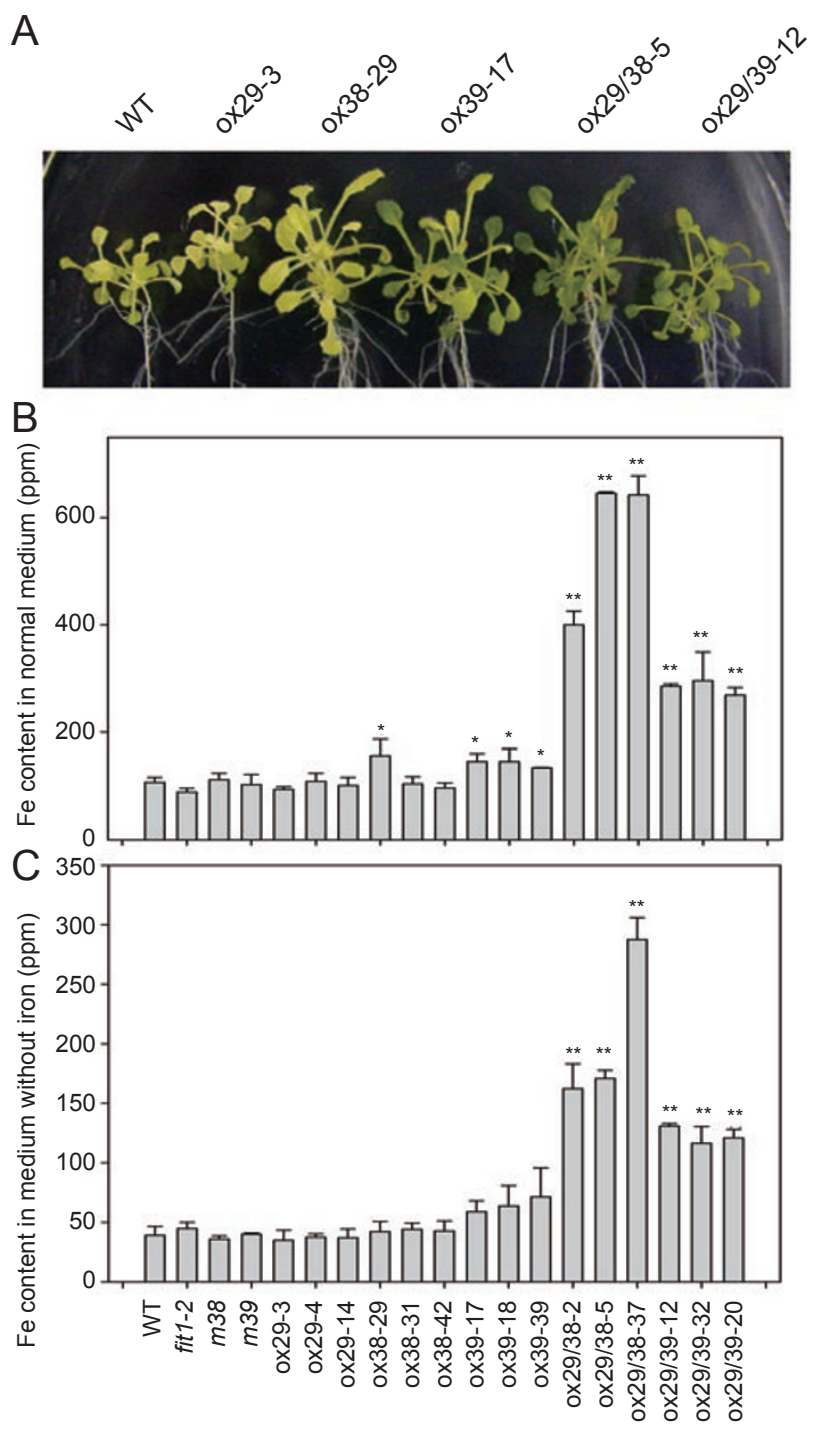

Figure 4 Phenotype and iron content of the plants overexpressing FIT, AtbHLH38, AtbHLH39, FIT/AtbHLH38 and FIT/AtbHLH39. (A) Phenotype of the overexpression lines on MS plates without iron supply for 2 weeks. (B-C) Iron contents in shoots of plants grown on medium supplied with $100 \mu \mathrm{M}$ (B) and $0 \mu \mathrm{M} F e(I I I)-E D T A(C)$ for 10 days. Results are means of 3 independent experiments. * and ** indicate significant differences at the level of $P<0.05$ and $P<0.01$, respectively. fit1-2, $m 38$ and $m 39$ represent the corresponding T-DNA insertion lines of FIT, AtbHLH38 and AtbHLH39; ox29, ox38 and ox39 stand for plants overexpressing FIT, AtbHLH38 and AtbHLH39, respectively; ox29/38 and ox29/39 are plants overexpressing FIT/AtbHLH38 and FIT/AtbHLH39, respectively.

in yeast cells (Figure 1). To examine whether this is the case in planta, FIT, AtbHLH38 and AtbHLH39 under the control of CaMV $35 \mathrm{~S}$ promoter were separately constructed into a binary vector and the plasmids were introduced into the Arabidopsis genome by Agrobacterium-mediated flora dip method [33]. Transgenic lines of ox29 (overexpressing 
FIT), ox38 (overexpressing AtbHLH38) and ox39 (overexpressing AtbHLH39), as well as of ox 29/38 (overexpressing FIT and AtbHLH38) and ox29/39 (overexpressing FIT and AtbHLH39) were generated to overexpress single or double genes. After germination and growing on MS plates for 7 days, the seedlings of the overexpression plants together with wild type were transferred onto MS plates supplemented with 0 or $100 \mu \mathrm{M}$ Fe-EDTA. The plants of ox 29 , ox38 and wild type began to exhibit chlorosis on the fourth day and the ox39 plants on the seventh day after transferring onto iron-deficient MS plates, and no chlorosis was observed in ox 29/38 and ox 29/39 plants even after 2 weeks (Figure 4A). Under the condition of iron sufficiency, all overexpression plants (ox29, ox38, ox39, ox29/38 and ox29/39) like wild type plants showed normal growth (data not shown). These results demonstrate that the plants cooverexpressing FIT with AtbHLH38 or AtbHLH39 are more tolerant to iron deficiency than wild type and the plants overexpressing FIT, AtBHLH38 or AtbHLH39 alone.

To determine the effects of loss- and gain-function of AtbHLH38 and AtbHLH39 as well as FIT on iron accumulation, 3 homozygous lines of each single (ox29, ox38, ox39) and double overexpression (ox29/38, ox29/39) plants, and each loss-of-function mutant (fit1-2, $m 38$ and $m 39$ ) were characterized. After germination and growth on MS plates for 7 days, the seedlings were grown on MS plates with and without iron supply for 10 days. Shoots were harvested and their iron content was measured by ICP-OES. While the iron content of the three single overexpression lines of ox39 was statistically significantly higher than wild type, the lines of ox 29/38 and ox29/39 had 2 to 5 times more iron than wild-type when grown under iron-sufficient conditions (Figure 4B). fit1-2 showed lower iron content in its shoots than wild type and no significant differences in iron content were observed among $m 38, m 39$, ox 29 and wild type under iron sufficiency. Under iron-deficient conditions, the shoot iron values of ox29/38 and ox29/39 were significantly higher (more than $300-500 \%$ ) than that of wild type (Figure 4C). The iron content of fit 1-2, $m 38$, $m 39$, ox 29 , ox38, and ox39 was not significantly different from wild type under iron-deficient conditions. From these data, we conclude that overexpression of FIT/AtbHLH38 or FIT/AtbHLH39 is able to promote iron uptake and accumulation in transgenic plants and results in plants more tolerant of iron deficiency.

\section{Overexpression of FIT/AtbHLH38 or FIT/AtbHLH39 con-} verts FRO2 and IRT1 to constitutive expression

To clarify why the overexpression plants accumulate more iron in their shoots, expression patterns of FRO2 and $I R T 1$ were characterized by northern blot analysis. The steady state levels of FIT, AtbHLH38 and AtbHLH39
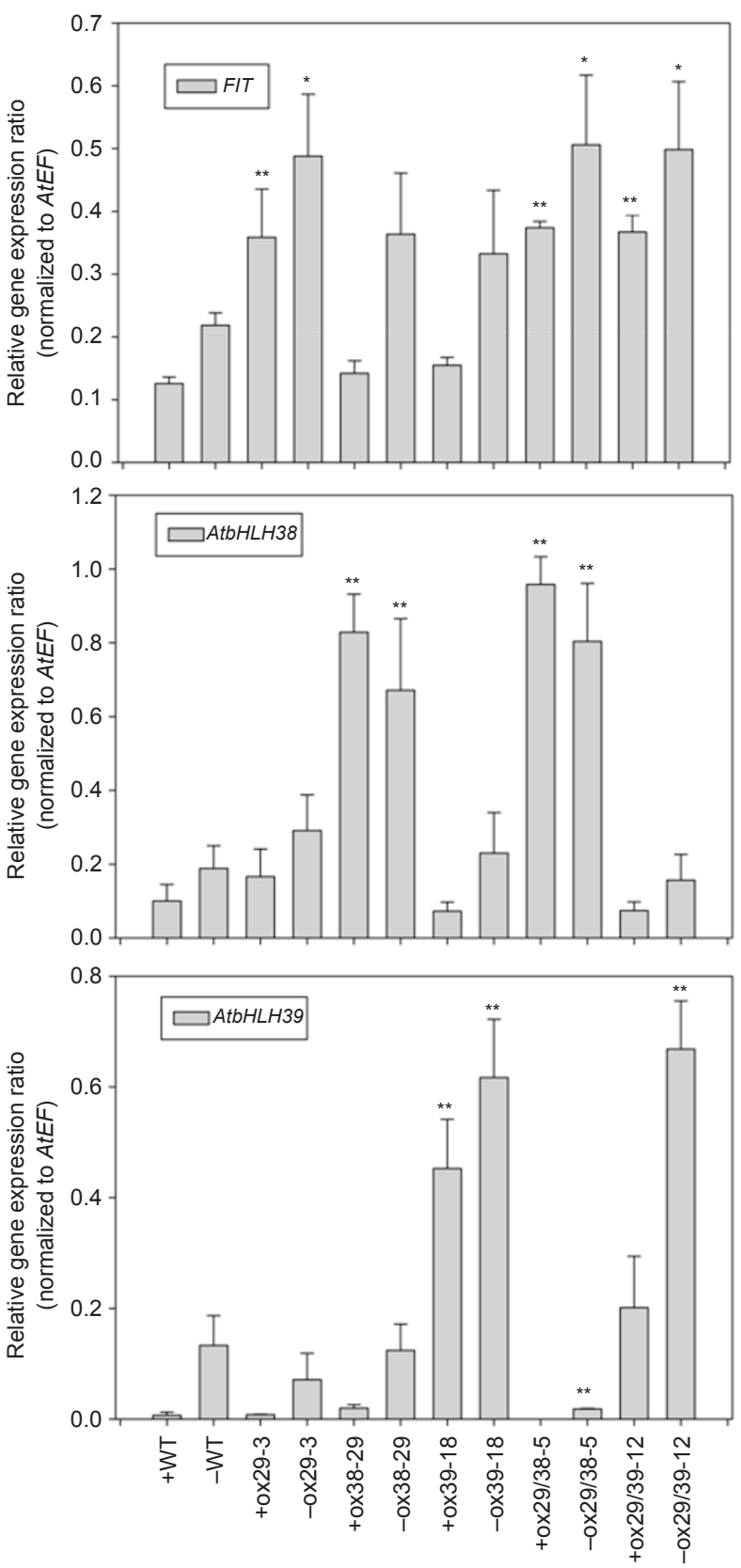

Figure 5 Expression profiles of FIT, AtbHLH38 and AtbHLH39 in the roots of ox29, ox38, ox39, ox29/38 and ox29/39. + and - mean the culture medium supplied with $100 \mu \mathrm{M}$ Fe(III)-EDTA and without iron. The relative gene expression ratio of FIT, AtbHLH38 and $A t b H L H 39$ was normalized to AtEF. Results are means of 3 independent experiments. Bars indicate standard error of mean. * and ** reveal significant differences at the level of $P<0.05$ and $P<0.01$ compared with wild type, respectively.

mRNAs in the lines were determined by multiplex RT-PCR analysis. All lines displayed enhanced expression of the corresponding gene(s) under both iron sufficient and defi- 


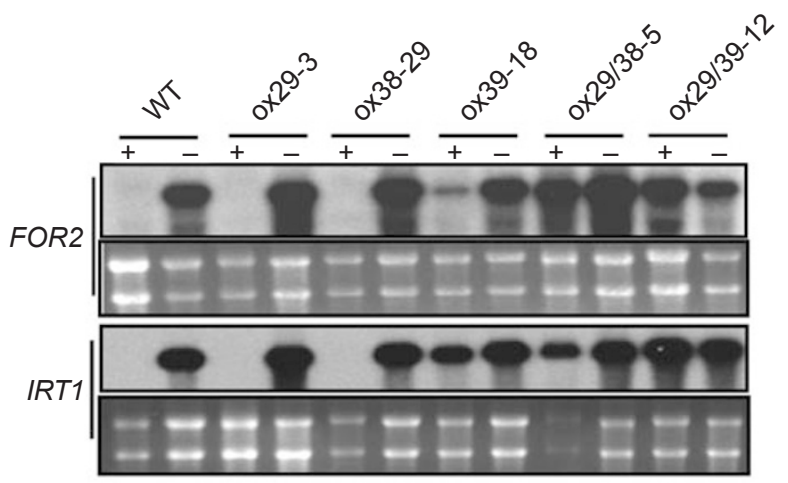

Figure 6 Northern blot analysis of $F R O 2$ and IRT1 expression in the roots of ox29, ox38, ox39, ox29/38 and ox29/39. + and - mean the medium with $100 \mu \mathrm{M} \mathrm{Fe}(\mathrm{III})-$ EDTA and without iron.

cient conditions (Figure 5). Although the expression of FIT and AtbHLH38 were obviously enhanced in roots of their corresponding transgenic plants regardless of iron status (Figure 5), in the single overexpression plants, all lines of ox 29 and ox38 displayed the same expression profiles of FRO2 and IRT1 as wild type under iron-sufficient and iron-deficient conditions (Figure 6). Interestingly, overexpression of FIT/AtbHLH38 or FIT/AtbHLH39 clearly converted the FRO2 and IRT1 to constitutive expression regardless of iron status, whereas the transcription of the two genes is only induced by iron deficiency in wild type. Additionally, IRT1 and FRO2 transcripts were also detected in ox39 plants under iron sufficiency although the mRNA abundance of the two genes was lower than that seen in ox29/38 and ox29/39 plants (Figure 6). This is fully consistent with the results of phenotypic and element analysis described above (Figure 4).

To further examine the enhanced expression of FRO2 and IRTI at protein level, one line carrying each single or double overexpression construct(s) was selected and ferric-chelate reductase activity was qualitatively and quantitatively analyzed under iron sufficiency conditions. Consistent with the mRNA results, the double overexpression plants showed more than two times higher ferric-chelate reductase activity compared to wild type and single overexpression plants (Figure 7A and 7B). Among the 3 single overexpression plants, the plant overexpressing FIT (ox29-3) displayed a higher ferric-chelate reductase activity than wild type whereas no significant differences were observed in the rest two single overexpression plants (ox38-29 and ox39-18) in comparison with wild type plants. Overexpression of $I R T 1$ does not result in protein accumulation when plants are grown under iron sufficiency [18]. To test whether the enhanced transcription of IRT1 caused by overexpression of FIT/AtbHLH38 or FIT/AtbHLH39 corresponded with an increased level of IRT1 protein, total proteins from the seedlings of ox29/38 and ox29/39 were extracted and analyzed by western blot analysis using an affinity-purified IRT1 peptide antibody [18]. IRT1 protein was found to accumulate in roots of ox29/38 and ox29/39 under both iron sufficiency and iron deficiency, whereas the accumulation of IRT1 protein in the control was observed in roots only under iron deficiency (Figure 7C). This result reveals that IRT1 expression was not regulated at the posttranscriptional level in the plants overexpressing FIT and either AtbHLH38 or AtbHLH39.
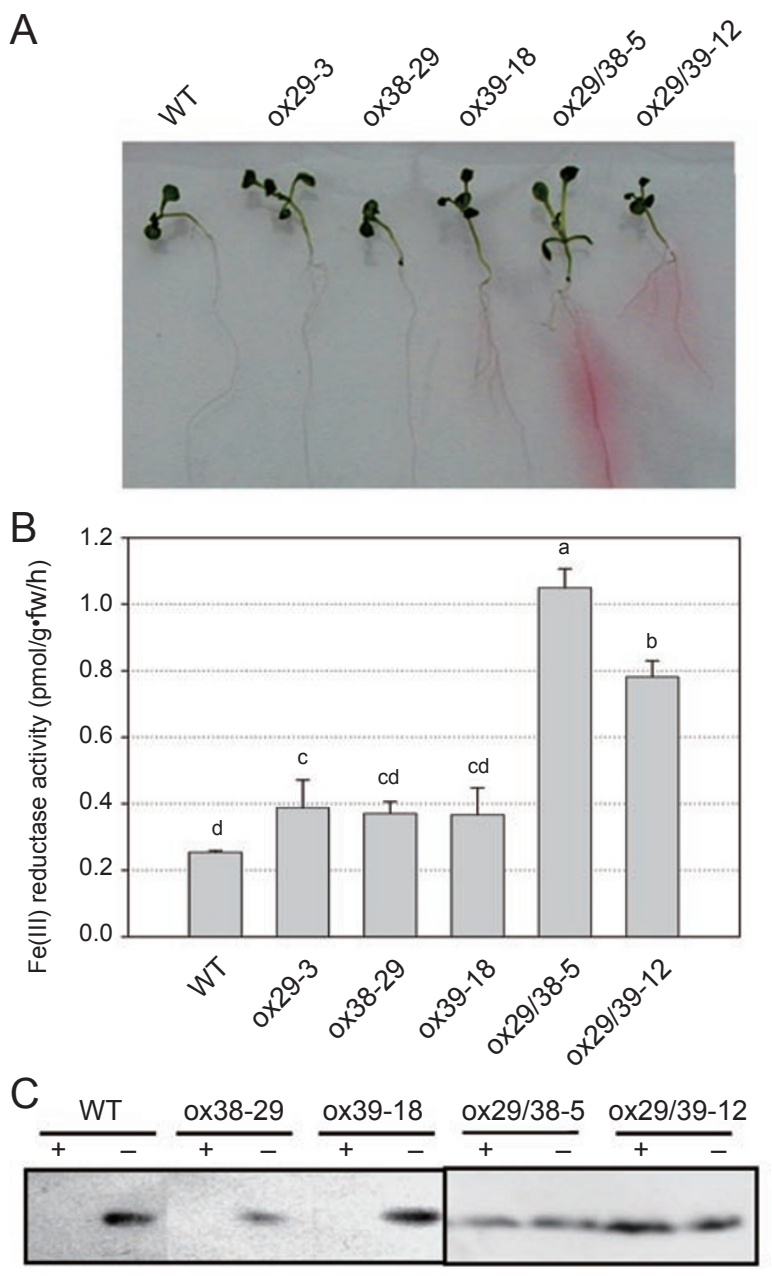

Figure 7 Ferric chelate reductase activity and Western blot analysis. (A-B) Qualitative and quantitative assays of ferric chelate reductase activities in roots of ox29, ox38, ox39, ox29/38 and ox29/39 grown under iron sufficiency, respectively. Red color around roots in (A) indicates iron reduction from $\mathrm{Fe}^{3+}$ to $\mathrm{Fe}^{2+}$ by ferric chelate reductase. Values are means of 3 independent experiments. Different letters indicate significant differences $(P<0.05)$ between the lines tested. (C) Western blot analysis of IRT1 protein in roots of overexpression lines of ox38-29, ox39-18, ox29/38-5 and ox29/39-12 as well as wild type grown under iron sufficiency $(+)$ and deficiency (-) conditions. 


\section{Discussion}

The bHLH proteins are a large family of diverse transcription regulators in both animal and plant cells. The formation of homodimers or heterodimers is an important feature of bHLH transcription factors. Based on their expression patterns, the bHLH transcription factors can be classified into two categories [34]. Members in the first category are usually broadly, or constitutively, expressed and can form homodimers. The expression of the members in the other category is often restricted to specific tissues or induced by specific physiological or environmental signals. They rely on the formation of heterodimers with the constitutively expressed bHLH proteins for their function.

In this work, we analyzed the targets and regulatory mechanisms of FIT, AtbHLH38 and AtbHLH39, whose expressions are regulated by iron status and involved in iron homeostasis. BiFC assay showed that AtbHLH38 and AtbHLH39 could not form homodimers in Arabidopsis cells. The results suggest that AtbHLH38 and AtbHLH39 belong to the second category of bHLH transcription factors which interact with other bHLH protein(s) to form heterodimers. Yeast two-hybrid assay and BiFC analysis in Arabidopsis cells demonstrated that AtbHLH38 and AtbHLH39 interacted with FIT, an essential regulator involved in the activation of the effective iron uptake system (strategy I) in Arabidopsis [21-24, 30]. The expression of FIT with AtbHLH38 or AtbHLH39 together in yeast cells activated GUS expression driven by either the $\mathrm{FRO} 2$ or the IRT1 promoter. Overexpression of FIT with AtbHLH38 or AtbHLH39 in Arabidopsis converted the expression patterns of FRO2 and IRT1 from induced to constitutive and the overexpression plants accumulated significantly higher iron levels in their shoots and displayed more tolerance against iron deficiency stress. Our data support that FIT interacts either with AtbHLH38 or AtbHLH39 and forms heterodimers (FIT/AtbHLH38 and FIT/AtbHLH39), directly functioning in controlling the transcription of the iron uptake genes FRO2 and IRT1, as well as in iron homeostasis of Arabidopsis.

AtbHLH38 and AtbHLH39 are located on the same chromosome adjacent to each other, and their proteins share $79.1 \%$ similarity [35]. AtbHLH39 might be a duplicated version of AtbHLH38 or vice versa. AtbHLH38 and AtbHLH39 belong to the same subgroup of the bHLH protein family in Arabidopsis [36, 31] and display similar expression patterns. AtbHLH38 and AtbHLH39 showed an interaction with FIT both in yeast cells and in plant cells. Knocking out one of the two genes did not disturb the expression patterns of FRO2 and IRTI, and the nullallele mutants of AtbHLH38 and AtbHLH39 had the same phenotype as wild type both under iron sufficiency and iron deficiency. These results suggest that AtbHLH38 and AtbHLH39 have similar functions in iron homeostasis and possess functional redundancy. However, higher GUS activity driven by the IRT1 promoter was detected in the yeast cells expressing AtbHLH39 than those expressing AtbHLH38, and the accumulation of IRT1 mRNA was also detected in roots of the plants overxpressing AtbHLH39, but not in the roots of the plants overexpressing AtbHLH38 under iron sufficiency (Figure 6). Furthermore, plants overexpressing AtbHLH39 accumulated more iron in their shoots than plants overexpressing AtbHLH38 (Figure 4B and $4 \mathrm{C}$ ). These indicate that AtbHLH39 might possess an additional function in controlling IRT1 expression. Considering that AtbHLH39 can not form homodimers in Arabidopsis cells (Figure 2G), it must be interacting with an unknown factor.

The ferric chelate reductase $F R O 2$ and the ferrous transporter IRTI are two major iron acquisition genes in Arabidopsis. The two genes are coordinately induced together by iron starvation and repressed following iron resupply [8]. Increasing mRNA levels by overexpressing one of the two genes results in accumulation of the corresponding mRNA in both roots and shoots under ironsufficient and iron-deficient conditions, but ferric chelate reductase activity and IRT1 protein are only detected and accumulated in iron-deficient roots $[8,18]$, suggesting that the expression of $F R O 2$ and $I R T 1$ are regulated at both the transcriptional and posttranscriptional levels. Interestingly, such posttranscriptional regulation of $F R O 2$ and $I R T 1$ was not observed in the plants overexpressing FIT/AtbHLH38 or FIT/AtbHLH39. Besides increased steady state levels of mRNA of FRO2 and IRT1 (Figure 6), increased ferric chelate reductase activity (Figure 7A and 7B) and IRT1 protein accumulation (Figure 7C) were observed in the roots of ox29/38 and ox29/39 plants under iron sufficiency. This might be explained by the coordinate elevation of $\mathrm{FRO} 2$ and IRTI mRNA via overexpression of the transcription factors and the resulting balance of FRO2 and IRT1 in vivo. It is also possible that AtbHLH38 and AtbHLH39, with or without FIT, could regulate as yet unknown factors involved in the posttranscriptional regulation.

The data presented here strongly support that AtbHLH38 and AtbHLH39 interact with FIT in regulating iron homeostasis in Arabidopsis. The three transcription factors function in the transcriptional regulation of the iron uptake genes such as FRO2 and IRT1. A model based on the results described here and reported previously is shown in Figure 8. Among the three transcription factors, FIT is a central, essential regulatory protein, which interacts with AtbHLH38 and AtbHLH39 to form heterodimers (FIT/AtbHLH38 and FIT/AtbHLH39) in root cells. The heterodimers directly activate or enhance the expression of the iron uptake genes, 


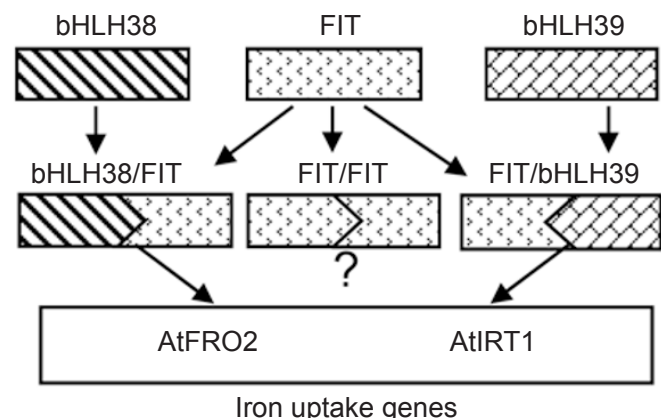

Figure $8 \mathrm{~A}$ regulation model of the transcription factors FIT, AtbHLH38 and AtbHLH39 on FRO2 and IRT1. FIT is an essential transcription factor for stimulation of the effective iron uptake system under iron deficiency stress in Arabidopsis. For the activation of FRO2 and IRT1 expression, AtbHLH38 or AtbHLH39 are required to form heterodimer with FIT.

such as FRO2 and IRTI, for effective iron acquisition. FIT can interact with itself in plant cells and form homodimer. However, the FIT dimer is not involved in controlling the transcription of FRO2 and IRT1 and might have other unknown functions based on facts that the expression of FIT alone did not activate the expression of GUS driven by either FRO2 or IRTI promoter in yeast cells and that overexpression of $F I T$ alone did not change the expression patterns of FRO2 and IRT1 in Arabidopsis. In conclusion, the illustration of the regulation mechanisms of FIT, AtbHLH38 and AtbHLH39 on their target genes FRO2 and IRT1 provides a new insight for studying the molecular mechanisms of iron uptake in strategy I plants and is very useful for design and breeding of iron-efficient crops for improving human iron nutrition.

\section{Materials and Methods}

\section{Plant growth conditions}

Seeds of Arabidopsis thaliana (ecotype Columbia), transgenic and T-DNA insertion lines were surface-sterilized with $10 \%$ commercial bleach for $10 \mathrm{~min}$ and then washed 3 times with distilled water. After vernalization at $4{ }^{\circ} \mathrm{C}$ in the dark for 2 days, the seeds were sown on plates with MS basal salt medium [37] supplemented with $2 \%$ sucrose, $0.6 \%$ phytogel and $\mathrm{pH} 5.8$. Plates were incubated at $23{ }^{\circ} \mathrm{C}$ with a $16 \mathrm{~h}$ light period for 7 days. The seedlings were then used for further analysis.

For the metal deficiency experiments, 7-day-old seedlings were transferred onto MS plates lacking either Fe, Zn or Mn. After growing for 4 days in the growth chamber with a $16 \mathrm{~h}$ light period, shoots and roots of plants were separately harvested and their total RNAs were extracted with Trizol reagent (Invitrogen) for studying gene expression profiles. For determination of iron contents in shoots, the 7-day-old seedlings were grown on MS plates with or without 100 $\mu \mathrm{M}$ Fe(III)-EDTA for 10 days. Subsequently, shoots were collected and their metal contents were measured by an Inductively Coupled
Plasma-Optic Emission Spectrometer (ICP-OES; Perkin Elmer, INC, USA) according to methods published previously [38].

\section{Identification of T-DNA insertion mutants}

The T-DNA insertion mutants (background Columbia) of AtbHLH38 and AtbHLH39 were obtained from NASC (The Nottingham Arabidopsis Stock Center) and are designated as $m 38$ and $m 39$, respectively. The T-DNA was inserted $42 \mathrm{bp}$ upstream of the AtbHLH38 (At3g56970) stop codon in $m 38$ (salk_108159), and 92 bp downstream of the AtbHLH39 (At3g56980) start codon in $m 39$ (salk_025676). Additionally, a T-DNA insertion mutant of FIT (fit12 [21]) was also used in this experiment. All the mutations were confirmed at genome level by PCR analysis with specific primers as described by Salk Institute Genomic Analysis Laboratory and at the transcriptional level by GeXP analysis (Beckman, Fullerton, CA) with gene-specific primers MRT29, MRT38 and MRT39 (Supplementary information, Table S1).

\section{Plasmid construction and plant transformation}

The coding sequences of FIT, AtbHLH38 and AtbHLH39 were amplified with gene specific primers (Supplementary information, Table S1) and cloned into pGEM-T Easy Vector (Promega, Madison, USA). After verification by DNA sequencing, the coding sequences of AtbHLH38 and AtbHLH39 were then cleaved by XbaI/SmaI and cloned into pBI121 to generate pBI121-35S::AtbHLH38GUS and pBI121-35S::AtbHLH39GUS constructs, whereas the coding sequence fragment of FIT was cut out and integrated at the sites $X b a \mathrm{I} / E c o R \mathrm{I}$ of pBI121 to generate pBI121-35S::FIT construct. In order to produce plants overexpressing AtbHLH38 and FIT or AtbHLH39 and FIT, the expression cassettes 35S::AtbHLH38GUS and 35S::AtbHLH39GUS were integrated into the vector pCAMBIA 1200 (CAMBIA, Australia) at the $\mathrm{XbaI} / \mathrm{SmaI}$ sites, generating pCAMBIA1200-35S::AtbHLH38GUS and pCAMBIA1200-35S: AtbHLH39GUS constructs. All the constructs were introduced into A. tumefaciens strain GV3101 by heat shock. The Agrobacterium clones were used to transform the ecotype Columbia through a floral dipping method [33]. Transgenic plants were selected on MS agar plates with corresponding antibiotic $(50 \mathrm{mg} / \mathrm{L}$ kanamycin sulfate or $60 \mathrm{mg} / \mathrm{L}$ hygromycin). Homozygous T3 lines were used for further analysis. The plants overexpressing FIT, AtbHLH38GUS and AtbHLH39GUS were named ox29, ox38, ox39, respectively.

To generate transgenic plants overexpressing FIT and AtbHLH38 or FIT and AtbHLH39, the pCAMBIA1200-35S::AtbHLH38GUS and pCAMBIA1200-35S::AtbHLH39GUS constructs were separately introduced into the homozygous ox 29 , which constitutively overexpresses FIT, by A. tumefaciens-mediated transformation. Transgenic plants were selected on MS agar plates containing $60 \mathrm{mg} / \mathrm{L}$ hygromycin. The plants overexpressing FIT and AtbHLH38 or FIT and AtbHLH39 were named as ox29/38 and ox29/39, respectively.

\section{Yeast two-hybrid analyses}

The yeast two-hybrid analysis was performed according to the protocol of the Hybri-Zap two-hybrid system as described by the manufacturer (Stratagene, CA, USA). The coding sequences of FIT, AtbHLH38 and AtbHLH39 as well as AtbHLH40 (as a negative control) were amplified with primers Y29, Y38, Y39 and Y40 (Supplementary information, Table S1) by RT-PCR from total RNAs and ligated into the T-easy vector and sequenced for sequence verification. Then, the sequence fragments of AtbHLH38, AtbHLH39 
and AtbHLH4O were cleaved by EcoRI/SalI and separately integrated into pBD-GAL4 to generate the plasmids pBD-AtbHLH38, pBD-AtbHLH39 and pBD-AtbHLH40. FIT as bait was cloned at the EcoRI/SalI sites of pAD-GAL4 plasmid (defined as pAD-FIT). The plasmids were transformed alone or in pairs into yeast strain YRG2. The positive clones were selected on synthetic minimal (SM) medium without leucine or/and tryptophan and confirmed by PCR analysis. The interactions of the bait protein FIT with AtbHLH38 and AtbHLH39 as well as the negative control AtbHLH40 were evaluated for expression of the reporter gene HIS3 with an assay to determine histidine prototrophy on SM plates lacking leucine, tryptophan and histidine, and for the expression of $L a c Z$ by assaying $\beta$-galactosidase activity with a filter lift assay as described by the manufacturer.

\section{Regulation analysis of FIT, AtbHLH38 and AtbHLH39 on} their target genes in yeast cells

The ferric reductase gene FRO2 and the ferrous transporter gene IRT1 are possibly the direct target genes of FIT, AtbHLH38 and AtbHLH39. For identifying these and the possible regulation model, a yeast assay system was designed using the yeast two-hybrid system. The promoters of FRO2 and IRT1 with 5'-BamHI and 3'-HindIII sites were cloned into pGEM T-easy vector from genomic DNA of wild type Arabidopsis. The promoters were then cleaved with SacI/HindIII and used to replace the $2 \times 35$ S promoter of pJIT166 plasmid (pGreen; http://www.pgreen.ac.uk). The GUS expression cassettes with FRO2 or IRT1 promoter and CaMV terminator $\left(\mathrm{P}_{\mathrm{FRO}}::\right.$ GUS:: $\mathrm{T}_{\mathrm{CaMV}}$ and $\mathrm{P}_{\mathrm{IRT}}:: G U S:: \mathrm{T}_{\mathrm{CaMV}}$ ) were cut out from the pJIT166 derivatives with $\mathrm{SacI} / \mathrm{XhoI}$, blunted, and integrated into the PmacI site of $\mathrm{pBD}-F I T$ and $\mathrm{pBD}-\mathrm{GAL} 4$ to generate yeast expression plasmids pBD-GAL4-P $\mathrm{FRO}_{2}: \because G U S, \mathrm{pBD}-F I T-\mathrm{P}_{\mathrm{FRO} 2}:: G U S, \mathrm{pBD}-\mathrm{GAL} 4-\mathrm{P}_{\mathrm{IRT} 1}::$ $G U S$ and $\mathrm{pBD}-F I T-\mathrm{P}_{\mathrm{IRT}}:: G U S$. The plasmids were then introduced into yeast strain YRG-2 alone or in pairs with pAD-AtbHLH38, pAD-AtbHLH39 and pAD-AtbHLH4O.

\section{$\beta$-glucuronidase (GUS) and $\beta$-galactosidase (X-gal) activity assay in yeast cells}

Filter lift assay of $L a c Z$ reporter gene activity was performed according to the manufacturer's instruction (Strategene; http://www. stratagene.com). In brief, yeast clones grown on plates with histidine were transferred to nitrocellulose paper, and lysed by 3 repeated freeze-thaws in liquid nitrogen for $10 \mathrm{~s}$. The thawed paper was placed onto two-layers of filter paper soaked in Z buffer with X-gal for $3 \mathrm{~h}$. A similar method was used to evaluate GUS activity, except the thawed paper was soaked with the GUS staining buffer described by Jefferson et al. [39] for $3 \mathrm{~h}$.

The method for quantitatively measuring GUS activity was adapted from Jefferson [39]. Briefly, yeast cells were collected by centrifugation at $8000 \mathrm{rpm}, 0.2 \mathrm{ml}$ GUS extraction buffer were added to the yeast pellet per $1.0 \mathrm{ml}$ medium, followed by vortexing and three freeze-thaws in liquid nitrogen. Debris was cleared by centrifugation at $10000 \mathrm{rpm}$, and $2 \mu \mathrm{l}$ of the resulting supernatant were added to $198 \mu 1$ Brandford reagent for protein quantification, and another $10 \mu \mathrm{l}$ were incubated with $90 \mu \mathrm{l}$ of GUS assay buffer at $37^{\circ} \mathrm{C}$ for $30 \mathrm{~min}$. At different time intervals, each reaction was stopped with $900 \mu \mathrm{l}$ of $0.2 \mathrm{M}$ sodium carbonate, and $10 \mu \mathrm{l}$ were used for methylumbelliferone (MU) quantification. All the protein and MU quantification was determined using a TECAN microtiter plate spectofluorimeter (TECAN, Zurich, Switzerland). The results calculated as $\mathrm{pmol} \mathrm{MU} / \mathrm{min} / \mathrm{mg}$ protein. Three individual experiments were carried out and the averages were calculated.

\section{Transient analysis of protein/protein interaction in Arabidop- sis protoplasts}

The coding sequences of FIT, AtbHLH38 and AtbHLH39 were amplified with primers BF29, BF38 and BF39 (Supplementary information, Table S1) and cloned into pX-nYFP and pX-cCFP vectors using the Gateway-compatible vector cloning system [32] to generate constructs of BiFC-FIT-nYFP, BiFC-FIT-cCFP, BiFCAtbHLH38-nYFP, BiFC-AtbHLH38-cCFP, BiFC-AtbHLH39-nYFP and BiFC-AtbHLH39-cCFP. Protoplasts were isolated from the leaves of 3 week-old Arabidopsis plants and transformed by the PEG transformation method according to the description of Sheen [40]. As a positive control, the plasmid pA7-YFP was introduced into the protoplasts. After incubation for 18-24 h, the protoplasts were assayed and fluorescence emission of YFP in living cells was observed under a Confocal microscope (Olympus, Japan). The fluorescence signals was observed at an excitation wavelength of $488 \mathrm{~nm}$ and emission wavelength of 506 538 nm, and the autofluorescence of chloroplasts was observed at excitation wavelength of $488 \mathrm{~nm}$ and emission wavelength of 664-696 nm.

\section{Multiplex RT-PCR analysis}

The primers used in the multiplex RT-PCR reactions are described in Supplemental Table S1. Each reverse primer is chimeric with the $5^{\prime}$ end containing a 19-nucleotide universal priming sequence (GTA CGA CTC ACT ATA GGG A) and the 3 ' end containing the gene-specific sequence. Each forward primer is chimeric with the 5 ' end containing a second 18-nucleotide universal forward priming sequence (AGG TGA CAC TAT AGAATA) and the 3 ' end containing the gene specific sequence. Each of the primer pairs was designed to yield PCR products 4 to $7 \mathrm{bp}$ apart, ranging from 149 to $389 \mathrm{bp}$. Primer design and multiplex optimization was performed using GeXP Express Profiler, primer design module (Beckman; http://www.beckman.com). The two universal primers, that are homologous to the $5^{\prime}$ ends of the chimeric primers with the forward universal primer carrying the D4 dye label, are included in the PCR reaction. The universal primers are included in the $5 \times$ GeXP PCR buffer.

The expression patterns of multiple genes were examined from each of the above samples using the GenomeLab GeXP Analysis System Multiplex RT-PCR assay (Beckman, Fullerton, CA) according the protocol described by Chen et al. [41]. The fragment results were analyzed on the express analysis module of the GeXP Genetic Analysis System. This software associates each PCR product with its corresponding gene and reports its peak area. The housekeeping gene AtEF (At5g19510) was used as the control. The gene expression data obtained from multiplex RT-PCR were normalized by dividing the peak area result of each gene by the peak area result of $A t E F$.

\section{Quantitative real time PCR}

After elimination of DNA contamination by treatment with RQ1 RNase-Free DNase (Promega, USA) at $37^{\circ} \mathrm{C}$ for $30 \mathrm{~min}$, the mRNAs were then converted to cDNAs using M-MLV reverse transcriptase (Invitrogen, USA) according to the manufacture's instruction. Quantitative Real-time PCR was performed using the Chromo4 real-time PCR detection system and OPTICON software version 3.0 (MJ research, USA). The gene-specific primers (Supplementary information, Table S1) and SYBR Green master mix (ABI, UK) were used according to the manufacturer's protocol in a final interaction 
volume of $20 \mu$ l. The Chromo 4 was programmed to $95^{\circ} \mathrm{C} 3 \mathrm{~min} ; 40$ cycles $\left(95^{\circ} \mathrm{C} 30 \mathrm{~s}, 55^{\circ} \mathrm{C} 30 \mathrm{~s}, 72^{\circ} \mathrm{C} 30 \mathrm{~s}\right), 72^{\circ} \mathrm{C} 5 \mathrm{~min}$ followed by a melting curve program $\left(65^{\circ} \mathrm{C}\right.$ to $95^{\circ} \mathrm{C}$ in increasing steps of $\left.1{ }^{\circ} \mathrm{C}\right)$. All reactions were repeated 4 times. The relative quantification values for each target gene were calculated by the $2^{-\Delta \Delta C}{ }_{T}$ method using AtEF as an internal reference gene for comparing data from different PCR runs or cDNA samples [42].

\section{Root ferric-chelate reductase activity assay}

Ferric-chelate reductase activity was determined for whole intact root systems as described by Waters et al. [4]. Ten-day-old seedlings, whose roots were rinsed with distilled pure water, were put on assay plates (1/2 MS Macro element, $100 \mu \mathrm{M}$ Fe(III)NaEDTA, $200 \mu \mathrm{M}$ BPDS (bathophenanthrolinedisulfonate), 0.6\% phytogel, $\mathrm{pH} 5.5)$ or submerged in assay solution $(200 \mu \mathrm{M} \mathrm{CaSO} 4,100 \mu \mathrm{M}$ Fe(III)NaEDTA, $200 \mu \mathrm{M}$ BPDS, 5 mm MES, pH 5.5). After $1 \mathrm{~h}$, an aliquot of the assay solution was removed and its absorbance was determined with a spectrometer at wave length $535 \mathrm{~nm}$. The $\mathrm{Fe}(\mathrm{II})$-BPDS concentration was calculated by using the extinction coefficient of $22.14 \mathrm{mM}^{-1} \mathrm{~cm}^{-1}$. The experiment was independently repeated three times.

\section{Northern blot analysis}

Ten $\mu$ g of total RNAs were denatured and electrophoresed on a 1.2\% 3-(N-morpholino)-propane-sulfonic acid/formaldehyde/agarose gel, and then blotted onto a nylon Hybond- $\mathrm{N}^{+}$membrane according to the manufacturer's instruction (Amersham, Buckinghamshire, UK). The probes, which were amplified by PCR using corresponding primers (Supplementary information, Table S1) from cDNA, were labeled with ${ }^{32} \mathrm{P}-\mathrm{dCTP}$. The hybridization, probe labeling and membrane washing were performed as described by Church and Gilbert [43]. Then, the membranes were exposed to Fujifilm (BASSR2025, Tokyo, Japan) in an X-ray imaging plate under $-70{ }^{\circ} \mathrm{C}$ for 1-3 days.

\section{Isolation of protein and immunoblot analysis}

Total proteins were prepared from the roots of Arabidopsis, which were grown on MS plates containing 0 or $100 \mu \mathrm{M} \mathrm{Fe(III)-EDTA} \mathrm{for}$ 4 days following the protocol described by Connolly et al. [18]. Ten $\mu \mathrm{g}$ of total proteins were separated by SDS-PAGE and transferred to polyvinylidene fluoride membranes by electroblotting. Membranes were blocked in $1 \times$ PBST $(0.1 \%$ Tween 20 in $1 \times$ PBS $)$ with $5 \%$ nonfat dry milk for $3 \mathrm{~h}$ at $37^{\circ} \mathrm{C}$. After washing in $1 \times$ PBST for 2 times ( 5 min each), the membranes were incubated overnight at $4{ }^{\circ} \mathrm{C}$ with affinity-purified IRT1 peptide antibody (1:1 000 dilutions in PBST and $1 \%$ nonfat dry milk). Subsequently, they were washed in $1 \times$ PBST 4 times (15 min each washing), and then incubated for $1 \mathrm{~h}$ with goat anti-rabbit IgG conjugated to horseradish peroxidase (1:5 000 dilution in $1 \times$ PBST and $1 \%$ nonfat dry milk). After washing in $1 \times$ PBST 4 times, chemiluminescence was performed using the Millipore Immobilon Western kit (Billerica, MA, BSA).

\section{Acknowledgments}

The authors thank Prof Mary Lou Guerinot (Department of Biological Sciences, Dartmouth College, Hanover, New Hampshire) for providing IRT1 peptide antibody and for the critical reading of the manuscript. We are also grateful to Drs Zhentao Lin and Yongfu Fu (Institute of Crop Sciences, Chinese Academy of Agricultural Sciences, Beijing) for providing the BiFC assay system and technical supporting. This work was supported by the National Natural Science Foundation of China (Grant nos, 30530460 and 30521001) and the Ministry of Science and Technology of China (Grant nos, 2005cb20904 and 2006AA10A105) and Chinese Academy of Sciences (Grant no. KSCX2-YW-N001) as well as by the Harvest Plus-China Program.

\section{References}

1 Marschner H. Mineral nutrition of higher plants. $2^{\text {nd }}$ Ed. London: Academic Press, 1995.

2 Halliwell B, Gutteridge JMC. Biologically relevant metal iondependent hydroxyl radical generation an update. FEBS Lett 1992; 307:108-112.

3 Robinson NJ, Procter CM, Connolly EL, Guerinot ML. A ferric-chelate reductase for iron uptake from soils. Nature 1999; 397:694-697.

4 Waters BM, Blevins DG, Eide DJ. Characterization of FRO1, a pea ferric-chelate reductase involved in root iron acquisition. Plant Physiol 2002; 129:85-94.

$5 \mathrm{Li} \mathrm{L}$, Cheng X, Ling HQ. Isolation and characterization of Fe(III)chelate reductase gene $\mathrm{LeFRO1}$ in tomato. Plant Mol Biol 2004; 54:125-136.

6 Wu H, Li L, Du J, Yuan Y, Cheng X, Ling HQ. Molecular and biochemical characterization of the $\mathrm{Fe}(\mathrm{III})$ chelate reductase gene family in Arabidopsis thaliana. Plant Cell Physiol 2005; 46:1505-1514.

7 Mukherjee I, Campbell NH, Ash JS, Connolly EL. Expression profiling of the Arabidopsis ferric chelate reductase (FRO) gene family reveals differential regulation by iron and copper. Planta 2006; 223:1178-1190.

8 Connolly EL, Campbell NH, Grotz N, Prichard CL, Guerinot ML. Overexpression of the $\mathrm{FRO} 2$ ferric chelate reductase confers tolerance to growth on low iron and uncovers posttranscriptional control. Plant Physiol 2003; 133:1102-1110.

9 Eide D, Broderius M, Fett J, Guerinot ML. A novel iron-regulated metal transporter from plants identified by functional expression in yeast. Proc Natl Acad Sci USA 1996; 93:5624-5628.

10 Vert G, Briat JF, Curie C. Arabidopsis IRT2 gene encodes a root-periphery iron transporter. Plant J 2001; 26:181-189.

11 Eckhardt U, Mas Marques A, Buckhout TJ. Two iron-regulated cation transporters from tomato complement metal uptake-deficient yeast mutants. Plant Mol Biol 2001; 45:437-448.

12 Bughio N, Yamaguchi H, Nishizawa NK, Nakanishi H, Mori S. Cloning an iron-regulated metal transporter from rice. J Exp Bot 2002; 53:1677-1682.

13 Ishimaru Y, Suzuki M, Tsukamoto T, et al. Rice plants take up iron as an $\mathrm{Fe}^{3+}$-phytosiderophore and as $\mathrm{Fe}^{2+}$. Plant $J$ 2006; 45:335-346.

14 Cohen CK, Fox TC. The role of iron-deficiency stress responses in stimulating heavy-metal transport in plants. Plant Physiol 1998; 116:1063-1072.

15 Henriques R, Jasik J, Klein M, et al. Knock-out of Arabidopsis metal transporter gene IRT1 results in iron deficiency accompanied by cell differentiation defects. Plant Mol Biol 2002; 
50:587-597.

16 Varotto C, Maiwald D, Pesaresi P, Jahns P, Salamini F, Leister D. The metal ion transporter IRT1 is necessary for iron homeostasis and efficient photosynthesis in Arabidopsis thaliana. Plant $J$ 2002; 31:589-599.

17 Vert G, Grotz N, Dedaldechamp F, et al. IRT1, an Arabidopsis transporter essential for iron uptake from the soil and for plant growth. Plant Cell 2002; 14:1223-1233.

18 Connolly EL, Fett JP, Guerinot ML. Expression of the IRT1 metal transporter is controlled by metals at the levels of transcript and protein accumulation. Plant Cell 2002; 14:1347-1357.

19 Ling HQ, Bauer P, Bereczky Z, Keller B, Ganal M. The tomato fer gene encoding a bHLH protein controls iron-uptake responses in roots. Proc Natl Acad Sci USA 2002; 99:13938-13943.

20 Bereczky Z, Wang HY, Schubert V, Ganal M, Bauer P. Differential regulation of nramp and irt metal transporter genes in wild type and iron uptake mutants of tomato. J Biol Chem 2003; 278:24697-24704.

21 Colangelo EP, Guerinot ML. The essential basic helix-loop-helix protein FIT1 is required for the iron deficiency response. Plant Cell 2004; 16:3400-3412.

22 Jakoby M, Wang HY, Reidt W, Weisshaar B, Bauer P. FRU (BHLH029) is required for induction of iron mobilization genes in Arabidopsis thaliana. FEBS Lett 2004; 577:528-534.

23 Yuan YX, Zhang J, Wang DW, Ling HQ. AtbHLH29 of Arabidopsis thaliana is a functional ortholog of tomato FER involved in controlling iron acquisition in strategy I plants. Cell Res 2005; 15:613-621.

24 Bauer P, Ling HQ, Guerinot ML. FIT, the FER-LIKE IRON DEFICIENCY INDUCED TRANSCRIPTION FACTOR in Arabidopsis. Plant Physiol Biochem 2007; 45:260-261.

25 Zhao T, Ling HQ. Effects of $\mathrm{pH}$ and nitrogen forms on expression profiles of genes involved in iron homeostasis in tomato. Plant Cell Environ 2007; 30:518-527.

26 Bailey PC, Martin C, Toledo-Ortiz G, et al. Update on the basic helix-loop-helix transcription factor gene family in Arabidopsis thaliana. Plant Cell 2003; 15:2497-2502.

27 Wang HY, Klatte M, Jakoby M, Bäumlein H, Weisshaar B, Bauer P. Iron deficiency-mediated stress regulation of four subgroup Ib BHLH genes in Arabidopsis thaliana. Planta 2007; 226:897908.

28 Vorwieger A, Gryczka C, Czihal A, et al. Iron assimilation and transcription factor controlled synthesis of riboflavin in plants. Planta 2007; 226:147-158.

29 Ogo Y, Itai $\mathrm{RN}$, Nakanishi $\mathrm{H}$, et al. The rice bHLH protein OsIRO2 is an essential regulator of the genes involved in Fe up- take under Fe-deficient conditions. Plant J 2007; 51:366-377.

30 Zhang J, Zhu HF, Liang H, et al. Further analysis of the function of AtbHLH29 in regulating the iron uptake process in Arabidopsis thaliana. J Integr Plant Biol 2006; 48:75-84.

31 Toledo-Ortiz G, Huq E, Quail PH. The Arabidopsis basic/helixloop-helix transcription factor family. Plant Cell 2003; 15:17491770 .

32 Gampala SS, Kim TW, He JX, et al. An essential role for 14-3-3 proteins in brassinosteroid signal transduction in Arabidopsis. Dev Cell 2007; 13:177-189.

33 Weigel D, Glazebrook J. Arabidopsis, a laboratory manual. New York: Cold spring harbor laboratory press, 2002:243-261.

34 Massari ME, Murre C. Helix-loop-helix proteins: regulators of transcription in Eucaryotic organisms. Mol Cell Biol 2000; 20:429-440.

35 Kang HG, Foley RC, Onate-Sanchez L, Lin C, Singh KB. Target genes for OBP3, a Dof transcription factor, include novel basic helix-loop-helix domain proteins inducible by salicylic acid. Plant J 2003; 35:362-372.

36 Heim MA, Jakoby M, Werber M, Martin C, Weisshaar B, Bailey PC. The basic helix-loop-helix transcription factor family in plants: a genome-wide study of protein structure and functional diversity. Mol Biol Evol 2003; 20:735-747.

37 Murashige T, Skoog F. A revised medium for rapid growth and bioassays with tobacco tissue cultures. Physiol Plant 1962; 15:473-497.

38 Herbik A, Bolling C, Buckhout TJ. The involvement of a multicopper oxidase in iron uptake by the green algae Chlamydomonas reinhardtii. Plant Physiol 2002; 130:2039-2048.

39 Jefferson RA, Kavanagh TA, Bevan MW. GUS fusions: betaglucuronidase as a sensitive and versatile gene fusion marker in higher plants. EMBO J 1987; 6:3901-3907.

40 Sheen J. Signal transduction in maize and Arabidopsis mesophyll protoplasts. Plant physiol 2001; 127:1466-1475.

41 Chen QR, Vansant G, Oades K, et al. Diagnosis of the small round blue cell tumors using multiplex polymerase chain reaction. $J$ Mol Diagn 2007; 9:80-88.

42 Livak KJ, Schmittgen TD. Analysis of relative gene expression data using real-time quantitative PCR and the 2(-Delta Delta C(T)) method. Methods 2001; 25:402-408.

43 Church G M, Gilbert W. Genomic sequencing. Proc Natl Acad Sci USA 1984; 81:1991-1995.

(Supplementary information is available at Cell Research's website.) 